\title{
Nonlinear imaging microscopy techniques as diagnostic tools for art conservation studies
}

\author{
George Filippidis, * Emilio J. Gualda, Kristalia Melessanaki, and Costas Fotakis \\ Institute of Electronic Structure and Laser, Foundation of Research and Technology-Hellas, P.O. Box 1527, \\ 71110 Heraklion, Greece \\ *Corresponding author: filip@iesl.forth.gr
}

Received November 29, 2007; revised December 18, 2007; accepted December 23, 2007; posted January 7, 2008 (Doc. ID 90321); published January 29, 2008

We present results of the implementation of three-photon excitation fluorescence (3PEF) and third harmonic generation imaging measurements for the precise and nondestructive detection of natural and synthetic varnish layers, which are used for the surface protection of painted artifacts. For this purpose, we employ as an excitation source a compact femtosecond laser operating at $1028 \mathrm{~nm}$. Two-dimensional images of the multilayer structures from different samples are depicted. The third harmonic signals show the interface between the different materials, when its refractive index mismatch is high enough. The depths of different layers of varnishes, presenting similar refractive index, are distinguishable with an axial resolution of $\sim 1 \mu \mathrm{m}$ by employing 3PEF measurements. () 2008 Optical Society of America

OCIS codes: $180.4315,190.1900,140.7090,110.0180$.

Determining the chemical composition of painting materials and understanding the painting techniques employed represent challenging issues in art history and conservation, with important practical implications. For example, provenance and authentication studies, as well as the choice of appropriate conservation strategies, may rely heavily on this type of information. Multicomponent and highly complex materials are usually employed, often undergoing changes due to various deterioration processes. Natural varnish layers (resins) are historically the most usual materials employed to protect painted surfaces from atmospheric pollution, oxidation, and improve the appearance of the artifact. However, varnishes suffer from progressive deterioration, due to aging and the continuous exposure to aggressive environmental conditions, emerging in the need of their removal [1-3]. On a typical painting more than one layer of varnish is employed, but mostly the outer layer is deteriorated, and it is required to be removed for rectifying the optical and aesthetic properties of the artwork. Similar needs exist for synthetic varnishes, which are used in many modern paintings for the protection of painted surfaces [2]. Precise thickness determination of those very complicated structures of multilayer varnishes is of high importance for the conservation of the artifact. Such information could ideally assist any restoration process, especially the cleaning, and help to avoid the damage of the exposed painted surface. To achieve this, various laser technologies have been applied for the measurement of varnish thickness, including laser-induced breakdown spectroscopy (LIBS) [2-5], and optical coherence tomography (OCT) [6]. Although these diagnostic techniques can assist laser based cleaning applications [4], they lack the resolution that is essential for the precise separation of different layers. Consequently, the cleaning procedure of an artwork is not fully controlled.

In this Letter we explore the feasibility of precise detection, measurement, and discrimination of differ- ent layers of varnishes by employing nonlinear imaging [three-photon excitation fluorescence (3PEF) and third-harmonic generation (THG)] measurements. This is the first time, to our knowledge, that nonlinear imaging measurements have been demonstrated for the precise separation (resolution in the order of $1 \mu \mathrm{m}$ ) of different layers of painting materials. This promising, unique, and nondestructive technique opens the road for the development of a variety of online diagnostic applications in art conservation and other fields.

The use of femtosecond lasers as the excitation sources have improved the resolution and 3D imaging capabilities of microscopy by multiphoton excitation, e.g., two-photon excitation fluorescence (2PEF) or 3PEF, and have also enabled new detection schemes, which exploit nonlinear excitation effects, e.g., second-harmonic generation (SHG) and THG. The basic principle underlying these techniques is that for tightly focused femtosecond laser pulses, the photon density is high enough to induce multiphoton absorption or other nonlinear (coherent) processes within the focal volume, providing intrinsic threedimensionality. "Out of focal plane" photobleaching and phototoxicity are thus dramatically reduced, diminishing damage of the studied object. These nondestructive imaging methodologies exhibit the ability to section deeper within samples, since the fundamental incident light lies in the infrared (IR) spectrum region. Furthermore, optical higher harmonic generation, including THG, does not deposit energy to the specimen due to its energy-conservation characteristics, providing minimal sample disturbance (e.g., thermal, mechanical side effects), which is desirable for art conservation studies.

Confocal and multiphoton excitation microscopy measurements are mainly used to visualize endogenous or exogenous fluorophores in biology $[7,8]$ and very recently have been employed for studies in the area of archaeology [9]. SHG imaging measurements, for the evaluation of corrosion of painted metals, 
have been demonstrated [10]. THG is generated near the interface between two media. The primary contrast mechanism in THG microscopy is related to subfocal volume inhomogeneities in the optical properties of the sample. Under tight focusing conditions, the extent of THG increases dramatically when the focus spans an interface between two optically different materials. This allows imaging based on THG to resolve otherwise transparent interfaces and inhomogeneities within the resolution of the confocal parameter and without the use of external dyes [11-13].

The laser source used for the purposes of this Letter is a diode-pumped $\mathrm{Yb}$-doped solid state laser (Amplitude Systemes) with a repetition rate of $50 \mathrm{MHz}$ operating at a central wavelength of $1028 \mathrm{~nm}$. The average power at the laser output is $1 \mathrm{~W}$, and the pulse duration is $200 \mathrm{fs}$. A tunable attenuator is used to control the power to avoid burning spots in the samples. The average laser power on the sample is $50 \mathrm{~mW}$ ( $1 \mathrm{~nJ}$ per pulse). The beam is introduced into an adapted microscope (Nikon Eclipse ME600D) with a two-lens arrangement served to expand the beam to fully fill the aperture of the objective lens (Nikon $50 \times$ NA 0.8 ). The lateral resolution on the focal spot is $785 \mathrm{~nm}$. A second objective lens (Carl Zeiss PlanApochromat $100 \times$ NA 1.4 oil immersion) is used to collimate the third harmonic forward generated signal. After passing through a $340 \mathrm{~nm}$ interference filter (Hoya U340), the signal is sent to a photomultiplier tube (Hamamatsu H9305-04) connected to a lock-in amplifier (SR810 Stanford Research Systems). The recording time is $1 \mathrm{~ms}$ in every step. The $3 \mathrm{PEF}$ signals are collected in the backward direction using a photomultiplier tube (PMT) (Hamamatsu R4220) connected to another lock-in amplifier. A short pass filter [(SPF) $650 \mathrm{~nm}$ (CVI Laser Corp.)] is placed at the photomultiplier input to cut off the reflected laser light. The focal position is controlled by a motorized stage (Standa 8MT167-100) with minimum steps of $1 \mu \mathrm{m}$, and the sample is scanned by two galvanometric mirrors (Cambridge Technologies). By detecting THG images in the transmission mode and 3PEF images in the reflection mode, our experimental apparatus allows the collection of two nonlinear optical signals, simultaneously.

Three different painting materials were studied. On round glass plates of $3.5 \mathrm{~mm}$ diameter and $\sim 45 \mu \mathrm{m}$ thickness (Marienfeld) different types of natural (mastic and colophony) and synthetic (vinavil, a polyvinyl acetate homopolymer) varnish layers were applied. Varnish solutions used were prepared according to established recipes (mastic was dissolved to turpentine, colophony in white spirit, and vinavil in water) and were casted to produce layers of even thickness. In all cases, pairs of two varnishes (natural or synthetic) were employed producing multilayer samples. All the experiments were performed by using fresh samples.

Figure 1 shows the absorption and emission spectra of mastic (solid curve), colophony (dashed curve), and vinavil (dotted curve) varnishes. The absorption [Fig. 1(a)] was measured with a UV/Vis spectrometer (Perkin Elmer-Lambda 950). It can be observed that
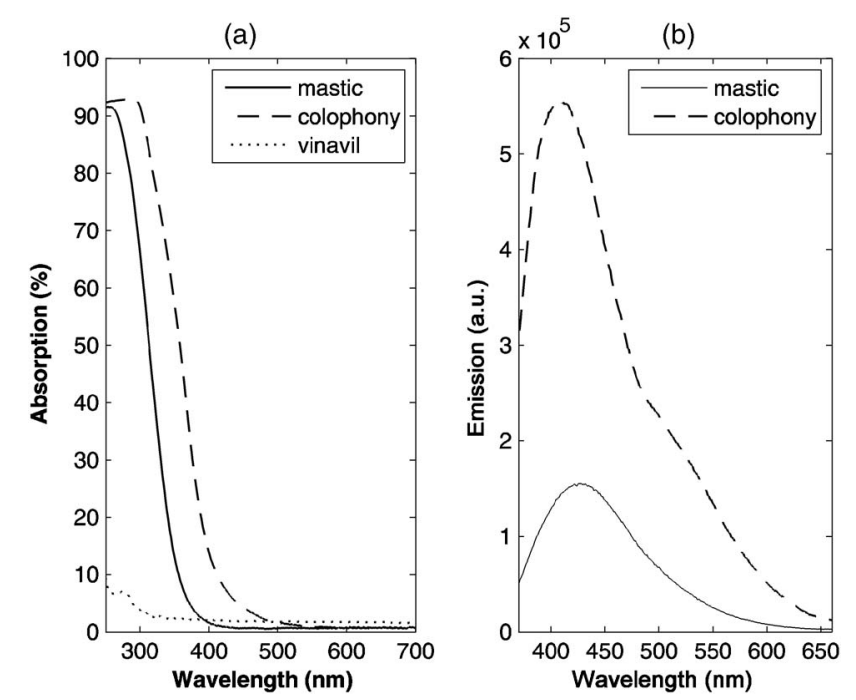

Fig. 1. (a) Absorption and (b) emission spectra of vinavil (dotted curve), mastic (solid curve), and colophony (dashed curve).

all the materials are transparent at $514 \mathrm{~nm}$ and have absorption maxima at UV wavelengths. The emission spectra were taken by using a fluoremeter (Jobin Yvon Horiba FluoroMax-P) with excitation wavelengths at 343 and $514 \mathrm{~nm}$. By employing the $343 \mathrm{~nm}$ wavelength for excitation, the emission spectra depict a maximum at $428 \mathrm{~nm}$ for mastic and a maximum at $408 \mathrm{~nm}$ for colophony [Fig. 1(b)]. When exciting at $514 \mathrm{~nm}$ no emission was detected, confirming that the origin of the fluorescence processes, due to three-photon excitation, at $343 \mathrm{~nm}$. Furthermore, as shown in Fig. 1(a) the absorption of vinavil (dotted curve) is less than $3 \%$ at $343 \mathrm{~nm}$ and, consequently, no fluorescence is observed.

Figure 2 shows two different samples combining layers of vinavil and mastic varnishes of different thickness. Through THG measurements [Figs. 2(a) (a)

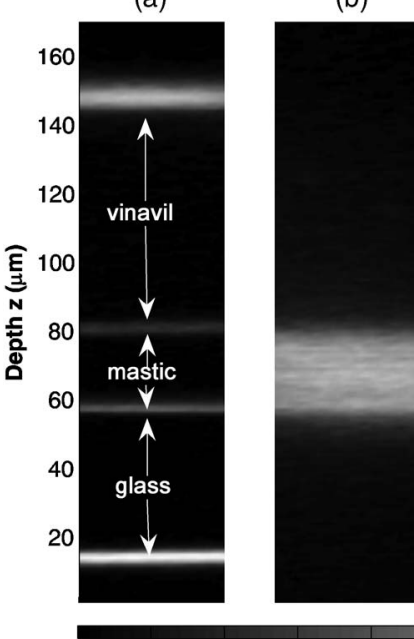

(c)

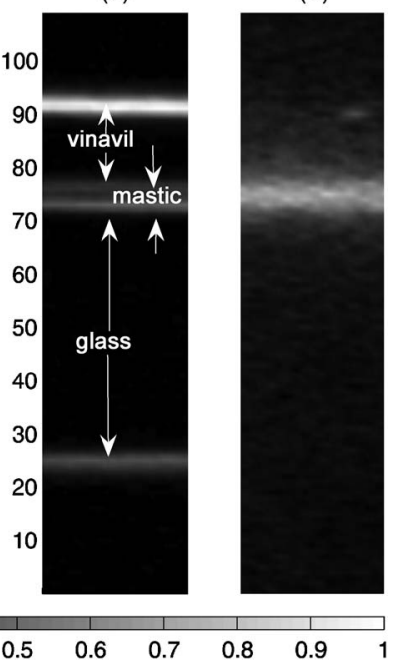

Fig. 2. Precise sectioning of a thick sample [(a) THG and (b) 3PEF] and a thin sample [(c) THG and (d) 3PEF] containing a layer of vinavil and a layer of mastic. The lateral dimension of the scanning area of the recorded images is $15 \mu \mathrm{m}$ with 300 points resolution. 
and 2(c)] four different layers can be distinguished, indicating the interface between the different media. The upper one corresponds to the air/vinavil interface and the lower one to the glass/air interface. The other two represent the vinavil/mastic and mastic/ glass interfaces, respectively. In sample 1 [Fig. 2(a)], the thickness of the vinavil layer is of $63 \mu \mathrm{m}$, whereas the mastic layer is of $22 \mu \mathrm{m}$. In sample 2 [Fig. 2(c)] thinner layers were created to check the resolution capabilities of the system. It is possible to distinguish a layer of mastic of $\sim 3 \mu \mathrm{m}$ thickness, while the layer of vinavil is of $16 \mu \mathrm{m}$. The thickness of the glass is always $\sim 45 \mu \mathrm{m}$. The detectable THG signals arising from the mastic/vinavil interface is due to the refractive index mismatch between the two media (1.536 for mastic and 1.487 for vinavil) [14]. Simultaneously, the 3PEF signals were measured in the backward configuration [Figs. 2(b) and $2(d)]$. It can be observed that signals only arise from the layer of mastic, providing complementary information in a single measurement. The measured thicknesses of the mastic samples correspond exactly to the thicknesses measured through THG measurements (22 $\mu \mathrm{m}$ for sample 1 and $\sim 3 \mu \mathrm{m}$ for sample 2 ).

Figure 3 presents the signal measured in a sample combining two different natural varnishes, colophony and mastic, with a refractive index of 1.548 and 1.536 , respectively [14]. The difference in the refractive index values between different resins is very low, so it cannot be distinguished by THG measurements [Fig. 3(a)]. However, due to the different absorptivity [20\% for mastic and 70\% for colophony, shown in Fig. 1(a)] two different levels of fluorescence signal are measured. As shown in Fig. 3(b), it is possible to achieve the precise discrimination of the thickness of each layer ( $22 \mu \mathrm{m}$ of mastic and $28 \mu \mathrm{m}$ of colophony)

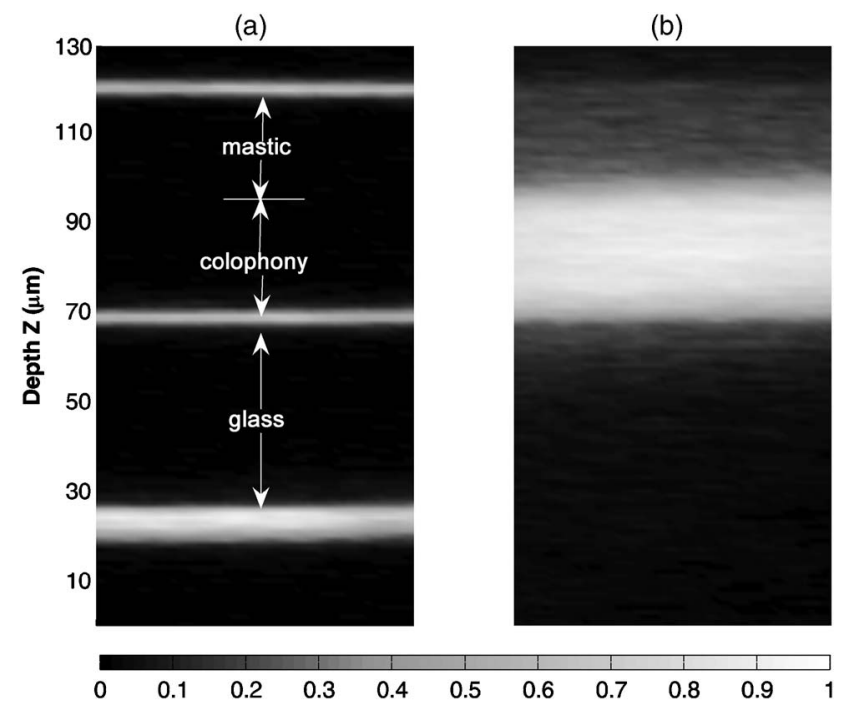

Fig. 3. Sections of a sample with two different layers of natural varnishes, mastic and colophony: (a) THG signal, (b) $3 \mathrm{PEF}$ signal. The lateral dimension of the scanning area is $15 \mu \mathrm{m}$ with 300 points resolution. through $3 \mathrm{PEF}$ imaging measurements. It is worth mentioning that all of the optical measured thicknesses of the materials were verified by measuring the samples thicknesses with a mechanical profilometer. In all cases the error bar is $<1 \mu \mathrm{m}$.

In conclusion, it has been shown that nonlinear image contrast modalities comprise a powerful diagnostic tool for art conservation studies. THG and 3PEF imaging measurements of natural and synthetic varnishes have been employed and bring forward a new approach for the precise determination of complex multilayer structures. Also, use of these innovative, nondestructive techniques that provide detailed information regarding materials thicknesses and spatial distributions would be helpful in a variety of applications, such as the identification of new and old varnish layers and the recognition of polymerized or oxidized varnish and more generally organic layers, facilitating the control of any cleaning interventions.

The authors thank the UV Laser Facility operating at Institute of Electronic Structure and LaserFoundation of Research and Technology-Hellas (IESL-FORTH) under the European Commission "Improving Human Research Potential" program (RII3-CT-2003-506350) and the Marie Curie Transfer of Knowledge project "Non Linear Imaging at Microscopic Level for Biological Applications (NOLIMBA)" (MTKD-CT-2005-029194).

\section{References}

1. R. J. Gettens and G. L. Stout, Painting Materials (Dover, 1966).

2. K. Melessanaki, C. Stringari, C. Fotakis, and D. Anglos, Laser Chem. 2006, 42709 (2006).

3. C. Fotakis, D. Anglos, V. Zafiropulos, S. Georgiou, and V. Tornari, Lasers in the Preservation of Cultural Heritage: Principles and Applications (Taylor \& Francis, 2006).

4. S. Georgiou, Z. Zafiropulos, D. Anglos, C. Balas, V. Tornari, and C. Fotakis, Appl. Surf. Sci. 127, 738 (1998).

5. D. Anglos, Appl. Spectrosc. 55, 186 (2001).

6. P. Targowski, M. Gora, and M. Wojtkowski, Laser Chem. 2006, 10647 (2006).

7. W. R. Zipfel, R. M. Williams, and W. W. Webb, Nat. Biotechnol. 21, 1369 (2003).

8. A. R. Woll, J. Mass, C. Bisulca, R. Huang, D. H. Bilderback, S. Gruner, and N. Gao, Opt. Lett. 31, 942 (2006).

9. I. G. Cormack, P. Losa-Alvarez, L. Sarrado, S. Tomas, I. Amat-Roldan, L. Torner, D. Artigas, J. Guitart, J. Pera, and J. Ros, J. Archaeol. Sci. 34, 1594 (2007).

10. J. Ying, F. Liu, P. P. Ho, and R. R. Alfano, Opt. Lett. 25, 1189 (2000).

11. J. A. Squier, M. Muller, G. J. Brakenhoff, and K. R. Wilson, Opt. Express 3, 315 (1998).

12. D. Debarre, W. Supatto, and E. Beaurepaire, Opt. Lett. 30, 2134 (2005).

13. S. W. Chu, S. Y. Chen, T. H. Tsai, T. M. Liu, C. Y. Lin, H. J. Tsai, and C. K. Sun, Opt. Express 11, 3093 (2003).

14. M. Elias, L. Simonot, M. Thoury, and J. M. Frigerio, Opt. Commun. 231, 25 (2004). 